NOTICIAS Y COMENTARIOS 



\title{
EL PLAN DE ORDENACIÓN DEL LITORAL DE GALICIA EN EL CONTEXTO DE LA GESTIÓN INTEGRADA DE LAS ZONAS COSTERAS
}

\author{
Isabel Ochoa Soto \\ isabel.ochoa.soto@gmail.com \\ Paula Pisos Parada \\ paulapisosparada@gmail.com
}

\section{LA NECESIDAD DE UNA ESTRATEGIA MARÍTIMA INTERNACIONAL Y NACIONAL. ESTADO DE LA CUESTIÓN}

Mientras que el papel de la atmósfera en las dinámicas climáticas resulta intuitivo y conocido por la mayoría de la población, facilitando la sensibilización social y el desarrollo de políticas al respecto, no sucede lo mismo con las aguas oceánicas, que pese a ocupar dos terceras partes de la superficie del planeta, no tienen apenas presencia en las agendas políticas de los gobiernos. Los mares ejercen una función esencial en la regulación energética de la Tierra, pues almacenan y transportan la mayoría de la energía que proviene del sol, de lo que resultan los distintos climas que caracterizan a cada una de las regiones planetarias. Esto obliga a ampliar la tradicional concepción que se tiene de los mares y océanos como enormes superficies de agua, funcionales sólo desde el punto de vista antrópico para el transporte de mercancías y como fuente de recursos pesqueros, y elevarlos a la categoría de vector energético de primer orden para la regulación del planeta, así como elemento clave dentro de la estrategia contra el cambio climático, al igual que ya se ha hecho con la atmósfera.

Esta situación de desconocimiento se manifiesta en la baja o nula conciencia ciudadana y en la escasez de medidas de gestión para proteger los océanos y las aguas continentales, tanto por parte de los gobiernos, como de los organismos internacionales. Prueba de ello es que frente a los esfuerzos internacionales, como el de Kioto, para disminuir la emisión de Gases de Efecto Invernadero (GEI) a la atmósfera, aún no existe ninguna política internacional que regule los vertidos al mar, quedando estos esfuerzos concentrados en normativa acotada a determinadas áreas geopolíticas como la UE o EEUU. Sin embargo, esta falta de regulación de la contaminación por vertidos no es el único problema que afecta a mares y océanos; a esto hay que agregar la total falta de 
ordenación del espacio marino, así como, de gestión de los recursos que acoge, independientemente del ámbito objeto de análisis .

Buena parte de los problemas que acechan al territorio marino, o Aquitorio (VV.AA, 2011), son consecuencia del cambio del modelo territorial que ha ido produciéndose paulatinamente, con más intensidad y rapidez en el último siglo. Esta transformación del modelo ha tenido una fuerte repercusión en el espacio marítimo, que se ha convertido en último término, en recolector de todas sus externalidades negativas. La presión urbanística de los últimos años, junto al intenso crecimiento demográfico de muchas áreas litorales, a lo que se suman nuevos usos como la acuicultura, la generación de energía mediante la creación de parques eólicos off-shore, o la modernización de las actividades tradicionales, como la pesca, que pierde su carácter sostenible para ganar productividad a corto plazo (tal y como exige el actual mercado global), son sólo algunos de los procesos nocivos que afectan al espacio marítimo. Todo ello ha provocado, además, una pérdida del carácter e identidad del área litoral, que ha tendido hacia una nueva imagen homogeneizada y banal, y a un claro deterioro de las áreas y recursos medioambientales y marinos (Fig. 1).

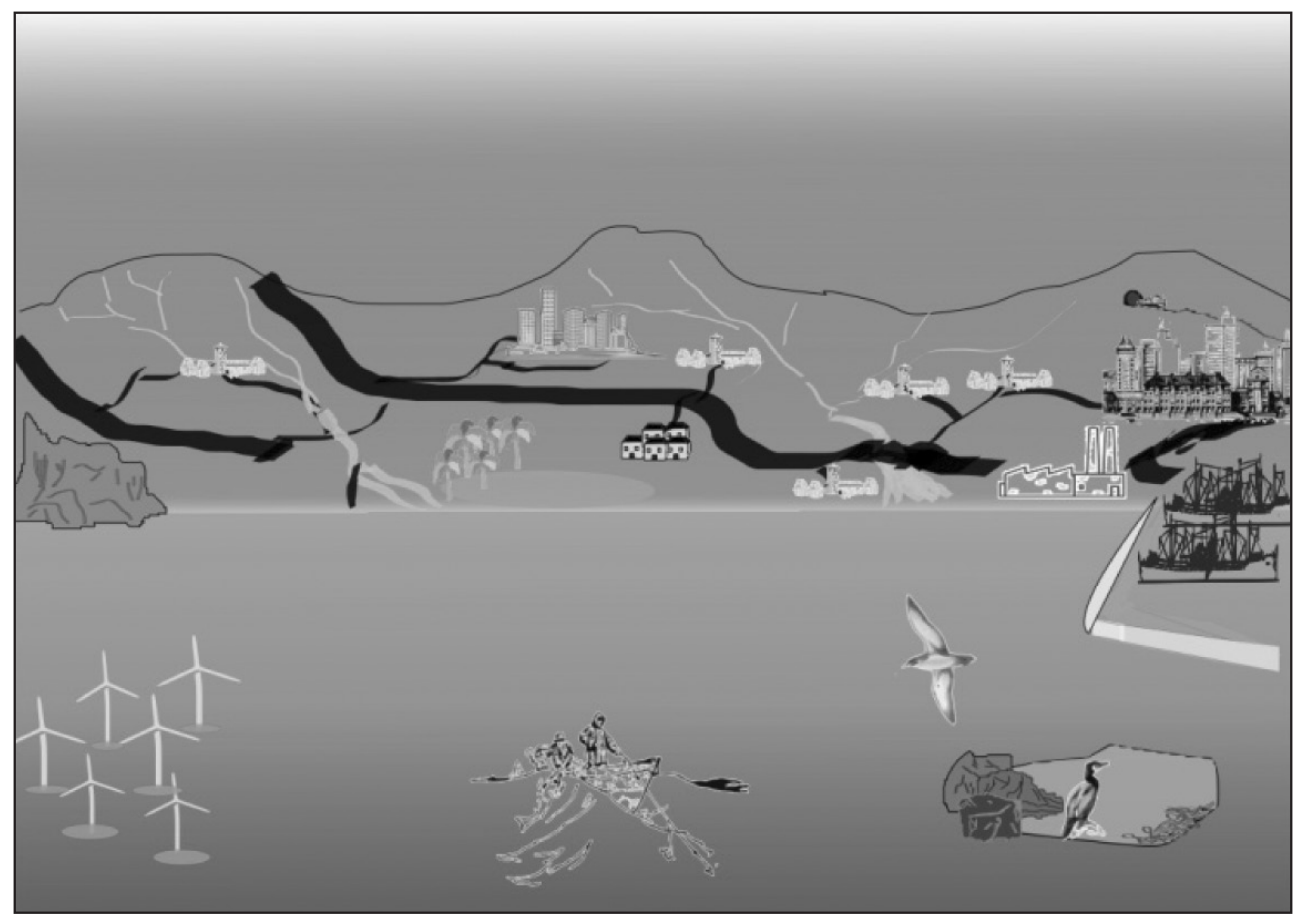

Fuente: Elaboración propia.

Ante estas dinámicas es preciso realizar una acción conjunta a escala transnacional que dé lugar a una ordenación del territorio costero, así como de las actividades que se desarro- 
llan en su ámbito. Los rápidos crecimientos urbanos, lejos de ajustarse a cualquier modelo racional, han tendido a ocupar buena parte de la línea de costa, obviando, por ejemplo, las consecuencia catastróficas que puedan derivarse de la subida del nivel del mar, efecto directo del calentamiento global, cuyo epicentro se encuentra en el vigente modelo socio-económico acontecido en tierra.

Desde la comunidad científica y desde distintas instancias intergubernamentales, conscientes de estos problemas, se ha trabajado en los últimos años para conocer y valorar el posible alcance de los mismos, y adoptar medidas para paliar sus consecuencias, especialmente en las escalas suprarregionales e internacionales. A tales escalas, el objetivo prioritario consiste en aunar esfuerzos en materia de ordenación y gestión del espacio marítimo y desarrollar una estrategia transnacional, basada en el compromiso y la cooperación de los distintos países implicados. Estos esfuerzos se han concretado, entre otras, en las siguientes iniciativas:

En el nivel internacional:

- Convenio Naciones Unidas sobre Derecho del Mar 1982 (CDM)

- Convención sobre la Diversidad Biológica, 1992

- Conferencia de Río de Janeiro, 1992

- Declaración de Washington sobre la protección del medio marino frente a las actividades realizadas en tierra, 1995

- Cumbre Mundial sobre desarrollo sostenible Johannesburgo, 2002

- Protocolo del Convenio de Londres, 2006

A escala europea:

- Plan de Acción del Mediterráneo, 1975 (en el marco del PNUMA)

- Convenio de Barcelona para la protección del Mar Mediterráneo, 1976 (Modificado 1995). Derivado de la anterior iniciativa.

- Carta Europea del Litoral, 1981

- Carta Europea de Ordenación del Territorio, 1983

- Programa demostración sobre gestión integrada de zonas costeras (GIZC), desarrollado por la Comisión Europea entre 1996 y 1999.

- La Recomendación 2002/413/CE del Parlamento Europeo y del Consejo de 30 de mayo de 2002 relativa a la aplicación de la gestión integrada de las zonas costeras en Europa.

- Convenio Europeo del Paisaje (CEP), 2000

- Directiva 2008/56/CE, por la que se establece un marco de acción comunitaria para la política del medio marino (Directiva Marco sobre la Estrategia Marina)

Esta nota centra su atención en la Estrategia Marítima Europea. Dicha Estrategia se refiere, de modo diferenciado, a tres ámbitos jurisdiccionales distintos (Aguas Jurisdiccionales, Territoriales e Interiores) (Fig. 2) y debe materializarse en estrategias de intervención diferenciadas tal y como se recoge en el diagrama adjunto. 


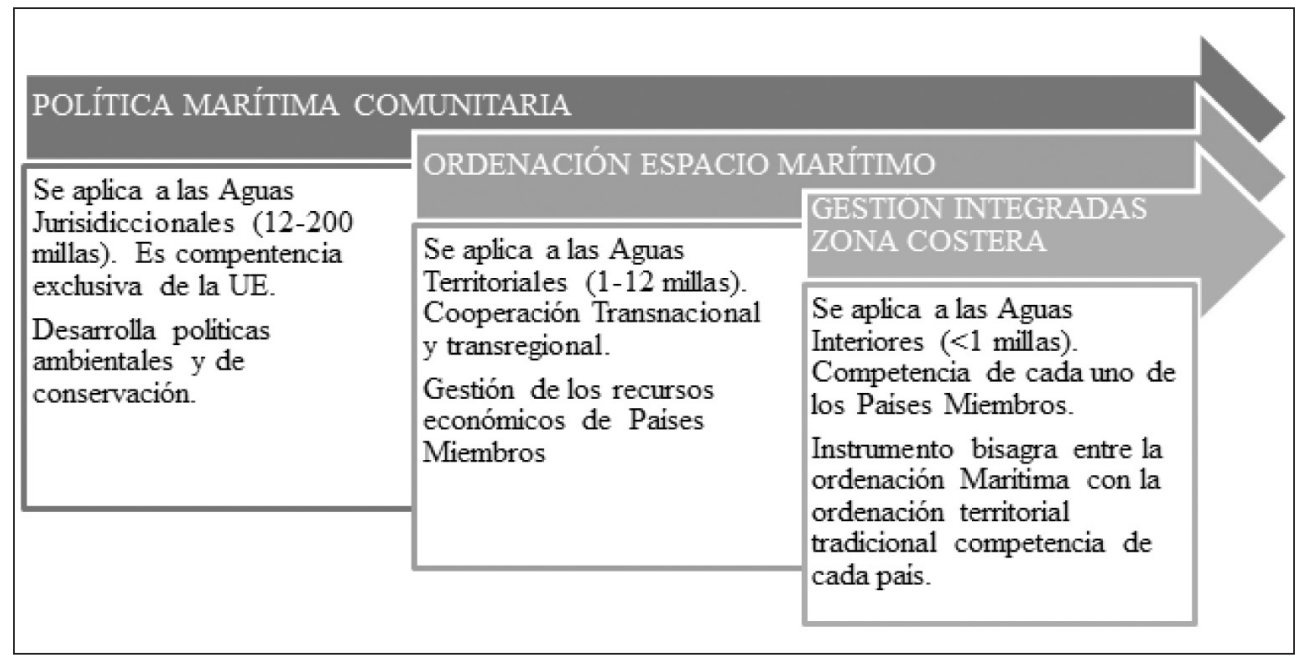

Fuente: Elaboración propia.

De estas tres políticas, son las de escala media y próxima al territorio las que están resultando más operativas; esto es lógico, pues los objetivos de la Política Marítima Comunitaria (PMC) son sólo alcanzables mediante la Ordenación del Espacio Marítimo (en adelante OEM) y una Gestión Integrada de las Zonas Costeras, en su acrónimo GIZC.

La OEM tiene por finalidad realizar una ordenación integral del territorio marino partiendo de un enfoque ecosistémico que se integre en las políticas y actividades con incidencia en este espacio, con objeto de velar por la utilización eficaz y sostenible tanto del Aquitorio como de sus recursos, de incentivar un mayor conocimiento e información del sistema marítimo-costero, y de generar un marco jurídico que permita el desarrollo económico de todos los países, coordinando los diversos intereses que son o puedan ser causa de conflicto. Esta ordenación, no obstante, se presume laboriosa y aún lejos de verse culminada por la dificultad que entraña.

Más urgente resulta, al menos desde el punto de vista de la PMC, la elaboración por parte de los estados miembros de la GIZC. Este instrumento de ordenación y planificación es la piedra angular que permite la integración de las estrategias europeas en materia de ordenación del océano en los habituales planes de ordenación del territorio litoral, como el Plan de Ordenación del Litoral de Galicia. La GIZC es, en suma, el camino para ampliar la visión que a escala de cada país se tiene del sistema costero-marítimo, permitiendo con ello superar la ordenación clásica del territorio litoral, de la que se ha hablado, y alcanzar una gestión más rica y completa, que integre el espacio marítimo y su interfase, sin la cual dicho sistema no tendría lugar.

A esta visión más amplia y compleja del concepto de litoral, se suma el ya mencionado enfoque ecosistémico. Dicho enfoque permite relacionar lo natural con la actividad antrópica, posibilitando un análisis y evaluación más profunda de los denominados impactos 
ambientales, y ampliando el horizonte ambiental más allá de la visión clásica del medio natural, sobre la que tradicionalmente se han focalizado los planes y proyectos oceánicosmarítimos. Eso es al menos lo que se pretende al tratar de cuantificar y valorar los efectos que, derivados de la actividad desarrollada por la moderna sociedad, inciden de forma directa o indirecta en dicho sistema.

Es responsabilidad y debiera ser competencia del planeamiento territorial, especialmente del referido a las áreas litorales, tener en cuenta estos aspectos. El litoral debe su origen y los principales rasgos de su carácter a la presencia del océano o el mar que lo modela y lo baña. El modelo territorial imperante hasta hace poco no ha considerado el mar como elemento clave del planeamiento, siendo a lo sumo un factor de atracción turística. Consecuencia de ello es, en parte, la actual configuración del litoral español, dominado por urbanizaciones que se aproximan cada vez más a la línea de costa mientras que, paradójicamente, desarrollan su crecimiento de espaldas al mar; actuando como si éste solo fuese un escenario o una frontera, que incluso, en los casos más extremos, se supera, creando nuevos espacios dentro del mar para edificar.

Es urgente, ante estas tendencias observadas y cada vez más extendidas, que los planes y actuaciones que se desarrollen en el ámbito litoral se impregnen de los principios y fines de la Estrategia Marítima y de los instrumentos de ella derivados, y que asuman mayor protagonismo en la ordenación y gestión de este espacio litoral-costero. En esa línea se han ido planteando los Planes de Ordenación del Litoral más recientes, siendo el último aprobado el de Galicia.

\section{APROXIMACIÓN AL POL DE GALICIA: METODOLOGÍA Y ASPECTOS INNOVADORES}

\section{Naturaleza jurídica del POL de Galicia}

El Plan de Ordenación del Litoral de Galicia, en adelante POL, es un instrumento de ordenación y gestión del espacio litoral, fruto de la diversa normativa que en materia territorial se ha ido desarrollando en los últimos años.

La Ley Orgánica 10/1995, de 23 de noviembre, de ordenación del territorio de Galicia, es la norma básica de regulación de los instrumentos y planes de ordenación y gestión del territorio. En su artículo 4 se reconocen las siguientes figuras de ordenación:

- Directrices de ordenación del territorio.

- Planes territoriales integrados.

- Programas coordinados de actuación.

- Planes y proyectos sectoriales.

- Planes de ordenación del medio físico.

La Ley 9/2002, de 30 de diciembre, de ordenación urbanística y protección del medio rural de Galicia otorga al POL, en su Disposición Transitoria Octava, la categoría de plan sectorial. Dicha naturaleza cambiará, pasando a ser un plan integrado, de acuerdo con lo establecido en el artículo 2 de la ley 6/2007, de 11 de mayo, de medidas urgentes en materia de ordenación del territorio y del litoral de Galicia, cuya regulación y función como plan integrado se recoge en el artículo 12 de la Ley 10/1995. 
Por último, el POL, a tenor de lo dispuesto en las Directrices de Ordenación del Territorio (DOT), da lugar a la delimitación de las Unidades de Paisaje, cuyo alcance normativo y de gestión se desarrollará en los futuros Catálogos y Directrices del Paisaje, conforme a lo dispuesto a la ley 7/2008 del 7 de julio, de protección del paisaje de Galicia.

\section{2. Ámbito y objetivos. El empleo del paisaje para la ordenación del litoral}

El ámbito de aplicación del POL se establece en el artículo 3.1 de la ley 6/2007, donde se define el litoral como «los 500 m medidos en proyección horizontal tierra adentro, desde el límite interior de la ribera del mar de los municipios que se relacionan en el anexo».

$\mathrm{Al}$ ámbito definido por la propia norma, se propone añadir, desde el instrumento de ordenación, un espacio pre-litoral que permita una visión más completa del sistema costero y, con ello, alcanzar una gestión verdaderamente integrada.

Como instrumento de Ordenación Integral, el POL de Galicia centra su objetivo en la ordenación del urbanismo siguiendo unos criterios de sostenibilidad y racionalidad, inexistentes hasta ese momento en el modelo de desarrollo imperante. Para ello el instrumento pone especial énfasis en la salvaguarda del medio ambiente y apuesta claramente por la protección de la franja litoral; al mismo tiempo es ambicioso tanto en la definición del ámbito geográfico de actuación, como por la cantidad de principios y criterios de distintas políticas y esferas que pretende integrar y coordinar.

Como base de la propuesta, el POL desarrolla un estudio integrador y global en torno al paisaje como unidad de análisis, ordenación y gestión del territorio. Este enfoque presenta grandes ventajas pues se aborda desde una visión holística del conjunto de elementos y dinámicas territoriales, superando así la clásica visión sectorizada y estanca que limita, entre otras cosas, la coordinación y colaboración entre las distintas políticas, aspectos sumamente importantes para cumplir los objetivos definidos en el plan y conservar la coherencia en el planeamiento territorial.

Dicha coherencia supone una mayor ventaja si se considera que va más allá de la coordinación intersectorial, pretendiéndose también una coordinación interadministrativa, posible sólo gracias al carácter fractal derivado de la propia concepción de paisaje, según la cual cualquier parte percibida del territorio es paisaje y, por tanto, en función de la escala, unos paisajes quedarán englobados en otros, al tiempo que un paisaje encierra multitud de ellos, resultando en conjunto un todo mayor que la suma de las partes. Esta cualidad se traduce en un documento, el POL, con un ámbito de aplicación regional, que se desagrega en unidades de paisaje, cuyos objetivos de calidad y contenido normativo se han de desarrollar en los futuros Catálogos y Directrices de Paisaje.

De esta manera, mediante este instrumento se pretende sentar las bases de una ordenación del territorio (pormenorizada en la medida en que se vayan desarrollando los instrumentos derivados a su vez del propio POL), con el paisaje como unidad básica de diagnóstico y propuesta. Asimismo, el paisaje se convierte, con esta concepción, en eje vertebrador del resto de políticas con incidencia territorial, pues articula la coordinación y coherencia con el propio territorio (superando el tratamiento homogeneizador que generalmente se ha llevado a cabo desde cada una de ellas), buscando como resultado un territorio y un paisaje diverso y de calidad. 
En definitiva, la ordenación enfocada desde el paisaje permite incorporar los criterios y estrategias de ámbito superior. Un ejemplo de ello es el papel que el propio POL se atribuye en la Gestión Integrada de las Zonas Costeras, recogiendo objetivos y principios generales de la misma y trasladándolos a un ámbito geográfico concreto; actuando, en suma, de bisagra entre las políticas de escala mayor y las políticas locales.

La propuesta se fundamenta en tres grandes principios: integración, equilibrio y cohesión. El primero de ellos hace especial énfasis a la integración de la urbanización (principal elemento de transformación del territorio) en el paisaje, es decir, en el carácter del litoral. El segundo principio, referido al desarrollo equilibrado de los territorios, pretende dirigir el crecimiento justificado hacia el interior, frente al modelo disperso y desbordado hacia el mar hasta ahora imperante, intentando con ello preservar la identidad y singularidad de la fachada litoral, e incrementar el esponjamiento urbano. El último de los principios, el de cohesión, se traduce en una propuesta de corredores ecológicos destinados a mejorar la permeabilidad natural, y en la elaboración de una estrategia de movilidad y transporte eficiente orientados no solo a mejorar la calidad de vida social, sino también, la competitividad económica.

\section{INSTRUMENTO DE INFLEXIÓN EN LA ORDENACIÓN DEL LITORAL. VALORACIÓN DE LAS PRINCIPALES CUESTIONES}

Habitualmente las notas referidas a los planes de gestión y ordenación del territorio tienen por objeto enfatizar los aspectos innovadores, y destacar los objetivos y principios que los sostienen y que dan sentido al trabajo realizado. Constituyen, por lo general, una aproximación básica, que los autores ofrecen al lector interesado, a modo de herramienta para que pueda enfrentarse a un documento que por su elevada complejidad y carga de contenido pudiera parecer a priori proceloso e inabarcable.

Sin embargo, pocas notas tienen por objeto definir la situación de los planes tras su implementación en la realidad territorial que con tanto detalle recogen y ordenan. Este es precisamente el objetivo prioritario de estas páginas. Por supuesto, no se pretende en modo alguno desmerecer el trabajo que ha supuesto la redacción del POL, cuyo carácter innovador en cuanto al enfoque empleado y metodología desarrollada está fuera de toda duda, así como la calidad técnica del equipo encargado de su redacción. No obstante, en esta ocasión se quiere hacer hincapié en cuatro de las cuestiones que se consideran claves dentro del POL, ya fuere por lo innovador de su planteamiento en un plan de estas características, o por poner de manifiesto problemas que en adelante habrá que resolver.

\section{La definición del ámbito del POL y su coordinación con la GIZC}

Los retos de la denominada Gestión Integrada de las Zonas Costeras (GIZC) se pueden sintetizar en dos: abordar de forma integrada la complejidad de las cuestiones territoriales y hacer efectiva la relación entre conocimiento y acción. (Farinós, 2010 y 2011). Esos dos retos constituyen la inspiración del POL; buen ejemplo de ello es el esfuerzo por desarrollar una visión ecosistémica dirigida a traspasar la barrera terrestre y adentrarse en la gestión de la costa como un binomio agua y tierra, con su respectiva interfase. 
El POL, innovador en su definición de litoral (ya que no se ajusta a lo establecido en la legislación que le da origen), trata de alcanzar una perspectiva más amplia extendiendo sus límites hacia el interior, abarcando un espacio que llama pre-litoral. Un ámbito mayor que posibilita un análisis de más rango y que permite identificar y comprender con mayor exactitud y profundidad los sistemas marítimos-terrestres, más aún, tiene como tarea pendiente extender los límites no sólo hacia el interior costero, sino también abrir la ordenación hacia el mar; siguiendo así uno de los criterios rectores de la Gestión Integrada de las Zonas Costeras (Fig. 3). El POL es sensible en su contenido a la problemática que aborda el GIZC, pero sin embargo, la inexistencia de una estrategia a escala de Galicia hace que este contenido resulte un tanto hueco, pues no se encuentra materializado en un instrumento concreto.

Figura 3

ÁMBITOS DE LAS POLÍTICAS E DE PROTECCIÓN DEL ESPACIO MARINO

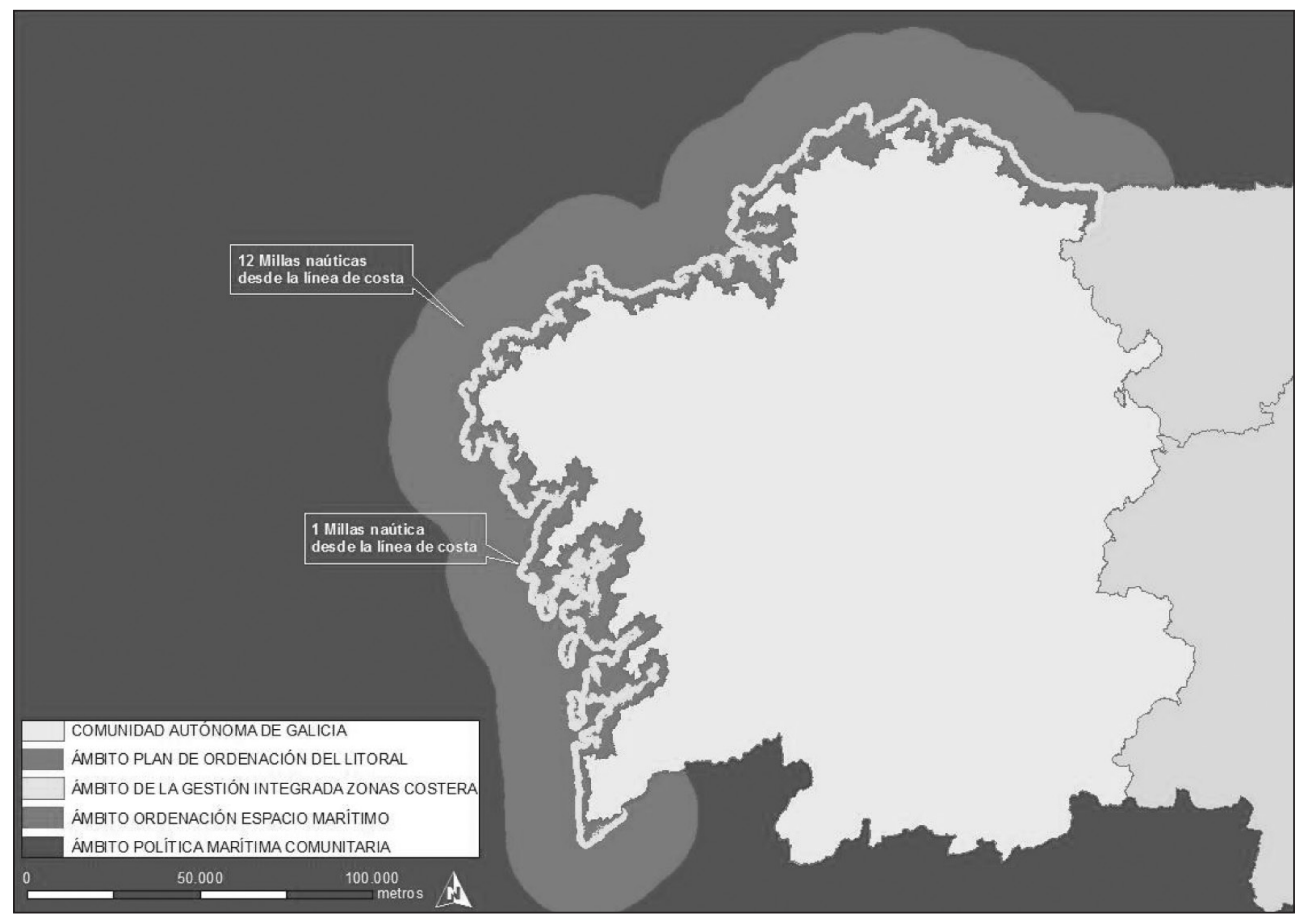

Fuente: Elaboración propia.

De esta manera el POL se presenta más bien como un instrumento dirigido a la ordenación que a la gestión del ámbito litoral, debiendo aludir y profundizar de forma expresa en la necesidad del desarrollo de la GIZC. No hay que olvidar que es recomendación de la UE que su elaboración y aplicación sea realizada lo antes posible por parte de los Estados miembros.

Dicha limitación, no obstante, tiene su razón de ser en el artículo 114 de la ley 22/1988, en el que se establece que la competencia autonómica sobre la ordenación del territorial y litoral alcanza solo el ámbito terrestre del dominio público marítimo terrestre (sin comprender el 
mar territorial y las aguas interiores); sin embargo, este artículo ha sido superado mediante la declaración de inconstitucional dictaminada por la Sentencia del Tribunal Constitucional $\mathrm{n}^{\mathrm{o}} 162 / 2012$, de 20 de septiembre. Con esta sentencia se abre el camino para el desarrollo de Planes de Gestión Integrada del Litoral por parte de la Xunta de Galicia, que podrá partir de cada una de las unidades de paisaje identificadas, elaborando un planeamiento más coherente e integrador con el conjunto del territorio.

\section{La Ordenación del Litoral: su análisis y diagnóstico}

A la hora de proponer la ordenación del litoral el POL distingue tres escalas o niveles de aproximación que van de lo regional hasta lo local (Fig. 4): Área Costera, Sector Costero y Unidades de Paisaje.

Figura 4

RELACIÓN ENTRE LAS DISTINTAS ESCALAS O NIVELES DE ORDENACIÓN

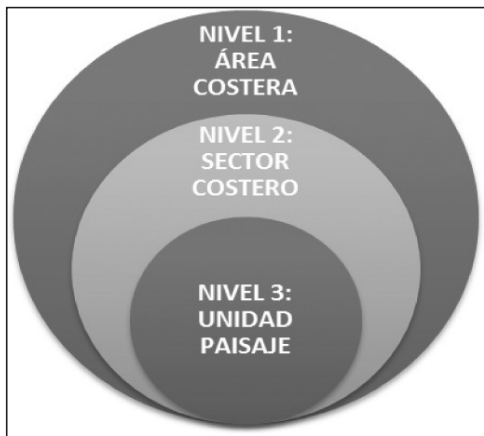

Fuente: Elaboración propia.

Las áreas costeras correspondientes al nivel 1 de la ordenación propuesta por el POL comprenden las grandes cuencas costeras representativas del litoral gallego.

El nivel 2 es resultado de la individualización de sectores característicos dentro de cada una de las áreas diferenciadas en el nivel 1.

El nivel 3 hace referencia a las diferentes unidades de paisajes reconocidas en cada uno de los sectores identificados en el nivel 2.

Esta diferenciación de escalas o niveles tiene la ventaja de que aglutina distintas ópticas del territorio en un mismo documento. De esta manera, desde la escala regional, la correspondiente al nivel 1, se puede desarrollar una planificación regional relacionada con las grandes áreas urbanas o infraestructuras. Mientras, la escala local que se emplea para los niveles 2 y 3 , permite una planificación pormenorizada del territorio, donde el paisaje se erige como eje de la planificación.

El documento realiza una apuesta clara por el desarrollo de una planificación a escala local, y por ello, no se detiene tanto como quizá debiera en los dos niveles superiores, especialmente cuando realiza su análisis y diagnóstico, lo que inhabilita la posibilidad de realizar una propuesta de ordenación a una escala del nivel 1 o 2 . 
Meritorio es, por otro lado, el esfuerzo en la identificación de las unidades de paisaje así como en su análisis y diagnóstico, aunque no los termina de desarrollar pues como bien se explica esa es tarea que corresponde a los Catálogos de Paisaje. Esto hace que, en realidad, el diagnóstico se plantee de forma sectorial y no de modo integral para los distintos ámbitos delimitados o para alguno de ellos al menos (ya sea el área, sector o unidad de paisaje). Con este diagnóstico se pierde parte del esfuerzo realizado anteriormente en la identificación y caracterización de cada uno de los espacios del litoral gallego, pues un diagnóstico sectorizado tiende inevitablemente hacia una homogeneización de los problemas y potencialidades de todo el litoral, en lugar de como es objetivo subyacente al trabajo, mostrar la diversidad territorial de la costa gallega (Fig. 5).

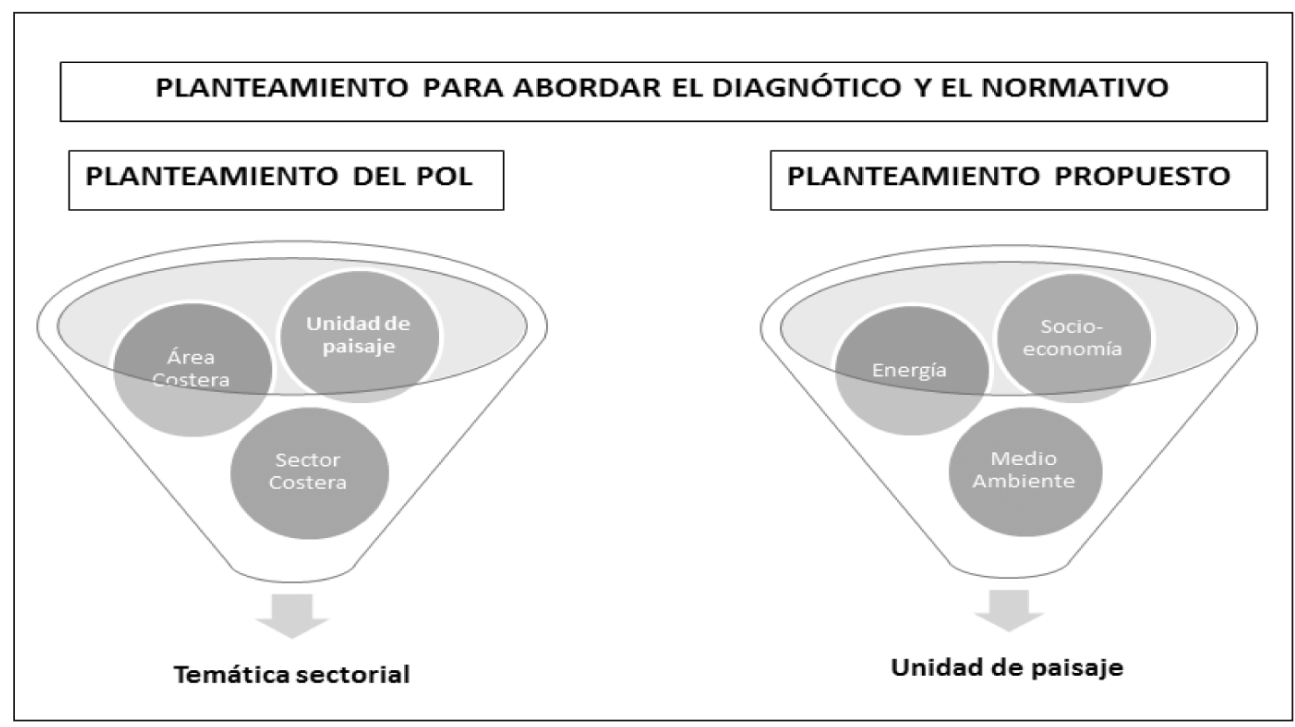

Fuente: Elaboración propia.

\section{La protección de los elementos singulares de la costa en el modelo territorial}

Dentro del espacio litoral quedan protegidas, además de la Red de Espacios Naturales Protegidos (ENP) de Galicia, las Áreas de Interés Paisajístico (AIP), las Áreas de Interés Geomorfológico (AIG) y las Áreas de Interés por Taxón (AIT), así como el Espacio de Protección de las Laderas (EPL). Todas estas figuras son propuestas y delimitadas por el POL. La superficie que se incluye dentro de cada una de las categorías se recoge en la siguiente tabla (Tabla 1): 
Tabla 1

SUPERFICIE OCUPADA POR CADA UNA DE LAS FIGURAS DE PROTECCIÓN ENUNCIADAS EN EL POL

\begin{tabular}{|c|c|c|c|c|c|}
\hline $\mathbf{A I P}\left(\mathbf{K m}^{2}\right)$ & $\mathbf{A I G}\left(\mathbf{K m}^{2}\right)$ & $\mathbf{A I T}\left(\mathbf{K m}^{2}\right)$ & $\mathbf{E P L}\left(\mathbf{K m}^{2}\right)$ & $\mathbf{E N P}\left(\mathbf{K m}^{2}\right)$ & $\operatorname{TOTAL}\left(\mathbf{K m}^{2}\right)$ \\
\hline 197 & 232 & 86 & 305 & 752 & 1.100 \\
\hline
\end{tabular}

Fuente: Elaboración propia.

Estas figuras se constituyen en los elementos base del modelo territorial, cuyo desarrollo compete al planeamiento municipal, siguiendo los criterios y principios que se recogen en el plan. Con ello se pretende la salvaguarda y puesta en valor, en todo el ámbito litoral, de aquellos elementos que dan carácter e identidad al territorio, siendo piezas claves a potenciar desde una escala local como elementos endógenos de desarrollo.

\section{EI POL de Galicia y la reforma de la Ley de Costas}

La Ley 22/1988 del 28 de julio de Costas es una norma veterana que marcó un hito en las políticas ambientales y sirvió de punto de inflexión en la protección del litoral frente al modelo urbanístico intensivo desarrollado en esta franja. Sus principales aportaciones se sintetizan en la delimitación del denominado Dominio Público Marítimo-Terrestre (DPMT), en el que queda prohibida la urbanización y deja pendiente de autorización los usos privativos de dicho espacio; asimismo, la ley establece unas servidumbres de protección, tránsito y acceso al mar; y el anuncio de una zona de influencia. Con esta ley se inicia un proceso de deslinde y amojonamiento de dicho espacio, que en el 2010 ya había sido aplicado al 93\% del litoral español.

Junto con este deslinde se ha procedido al desmantelamiento de numerosas urbanizaciones y viviendas que con la redacción de la ley quedaron declaradas como ilegales, existiendo, no obstante, conocidos casos en que la ley no se ha llegado a aplicar por lo complicado del proceso jurídico que cada una de estas actuaciones implica.

La revisión realizada en el año 2012 ha supuesto, sin embargo, un cambio significativo de dichos objetivos y fines. Las modificaciones introducidas flexibilizan la presencia urbanística en el litoral, abriendo una vía para paralizar los deslindes y derribos de las viviendas construidas irregularmente en el DPMT (espacio cuya extensión, a partir de la aprobación de la modificación, queda particularmente limitada en las rías y zonas de dunas); así mismo se prolongan las concesiones de determinadas actividades y se excluyen determinados núcleos como parte del DPMT sin justificación alguna. Todos estos cambios parecen conducir a la restitución de un modelo urbano contrario a los principios que inspiró originalmente a la ley.

El POL, como instrumento de ámbito regional que es, se encuentra sometido a las disposiciones establecidas por un instrumento de rango superior, como es la Ley de Costas. Según esta disposición jerárquica, el POL no puede transgredir los grados de protección establecidos en la ley 22/1988, cuestión que se vuelve especialmente conflictiva ante las modificaciones introducidas recientemente en dicha ley.

Una de las principales modificaciones con mayor afección sobre el POL es la referida al apartado 3 del artículo 23, que señala que «(...) En las riberas de las rías la extensión de esta 
zona podrá reducirse por la Administración del Estado de acuerdo con la de la Comunidad Autónoma y Ayuntamiento correspondiente, hasta un mínimo de 20 metros, en atención a las características topográficas o hidrográficas, a la anchura de sus cauces y a la distancia de la ribera de la ría respecto del límite interior de la ribera del mar».

Con esta nueva interpretación de la ley, las rías dejan de tener un espacio de protección de $100 \mathrm{~m}$. como de la normativa inicial se deducía. Esa iniciativa supone una importante desprotección de dicho espacio, que podría ser susceptible de urbanización, con los severos impactos que ello implica en la calidad del litoral, principal meta que persigue el POL.

Otra cuestión, quizá más relevante, es la paralización de los deslindes y derribos de las casas construidas con carácter irregular, ubicadas en el Dominio Público Marítimo-Terrestre, ante las que el POL no puede intervenir. Esto contraviene el alcance de los objetivos de esponjamiento del litoral y crecimiento hacia el interior establecidos por el POL, ya que no encuentran cobertura en la normativa de rango superior, que incluso, flexibiliza y, por tanto, tolera y hasta propicia la situación urbanística existente en la costa, contra la que el Plan pretende intervenir.

Ante estos cambios legislativos, el papel normativo del POL en la gestión de la costa parece cobrar un nuevo protagonismo, pues mientras que si de un lado no pueden ser sus disposiciones menos restrictivas que la normativa de rango superior, sí puede en cambio, completar o ampliar el horizonte de la misma. Este hecho resulta particularmente interesante en el caso que nos ocupa, pues ante la nueva flexibilización que ha adquirido la ley de costas el POL puede ejercer de amortiguador.

Otro punto fuerte de la normativa del POL y de su propia escala de aplicación es su capacidad diferenciadora del territorio, con una normativa específica según la naturaleza de cada uno de los paisajes litorales. Dicha normativa, además, permite superar la concepción exclusivamente geométrica de protección ambiental y adopta un enfoque ecosistémico e integrador de todas las dinámicas y facetas del territorio. Unido a lo anterior se encuentra un proceso participativo que acompaña a este trabajo en todas sus fases, lo que garantiza en parte que su normativa se encuentre respaldada por la población, lo que facilita su implementación y aceptación social, y lo refuerza como instrumento acorde con los principios de nueva gobernanza del territorio.

\section{TABLA DE CONCLUSIONES}

El POL es un instrumento altamente completo e innovador, con un marcado carácter didáctico para aquellos profesionales interesados en la ordenación y gestión del litoral. La experiencia pionera en la ordenación paisajística del litoral que representa el POL deja un balance de puntos fuertes a potenciar e imitar, y otros, los menos, que deben ser revisados para mejorar en futuras experiencias. En la siguiente tabla (Tabla 2) se presenta una síntesis de esos aspectos, que ya han sido tratados de forma más extensa a lo largo de la nota. 


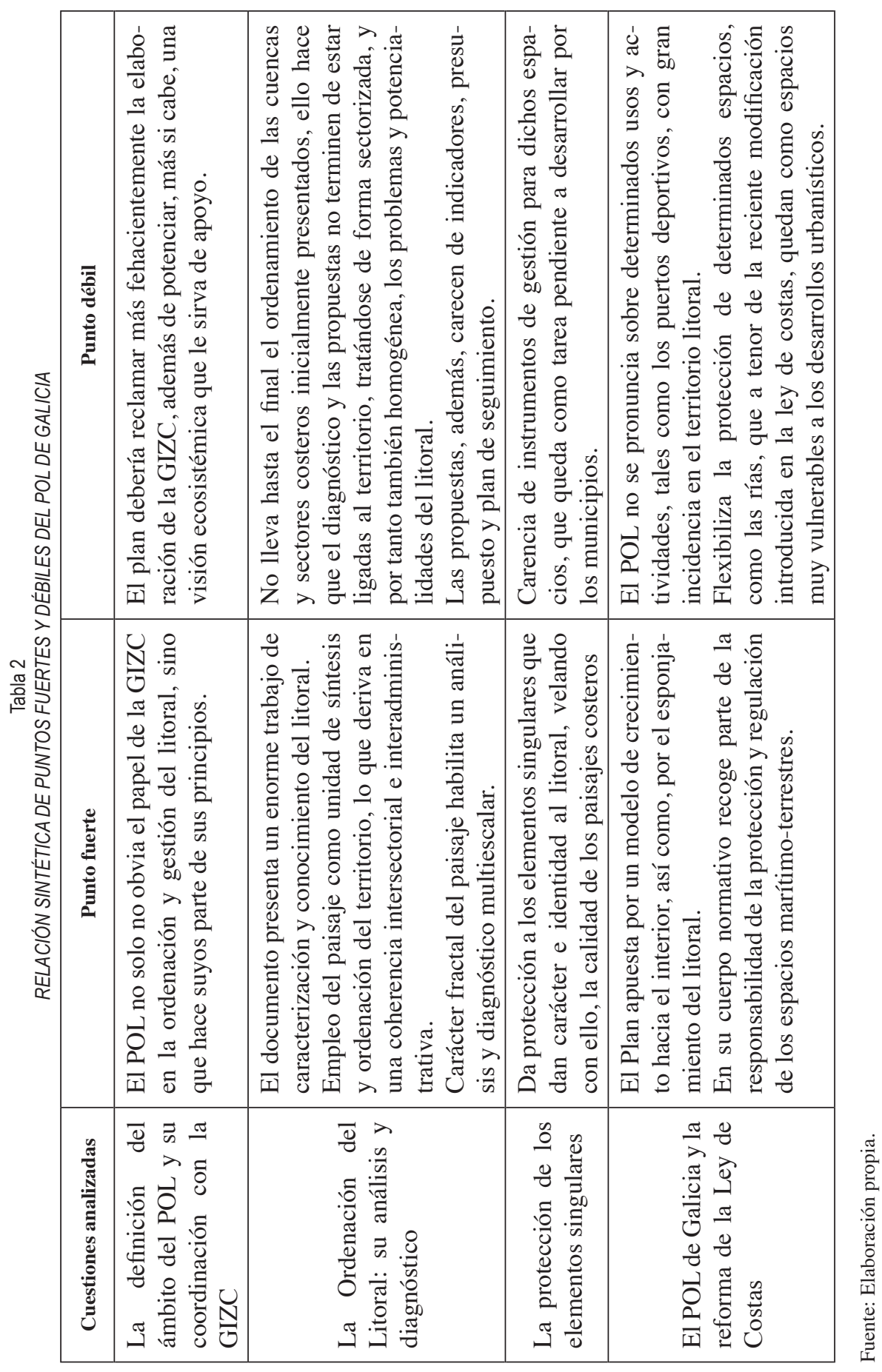




\section{BIBLIOGRAFÍA}

BOROBIO SANCHIZ, M. y GARCÍA GARCÍA, M. (2012): «Plan de Ordenación del Litoral de Galicia. Nuevas miradas para la ordenación territorial y la gestión del paisaje». Ciudad y Territorio, $\mathrm{n}^{\circ} 171$, pp. 171-177.

COMISIÓN EUROPEA (2007): Comunicación COM (2007) 308 final. Informe al Parlamento Europeo y al Consejo: evaluación de la gestión integrada de zonas costeras (GIZC) en Europa, de 7 de junio de 2007, Bruselas, 11 pp.

FARINÓS DASÍ, J. y CORTÉS TOVAR, M.C. (2010): «Planificación gobernanza del espacio litoral valenciano. Todavía lejos de una verdadera GIZC»Cuadernos de Geografía, $\mathrm{n}^{\circ} 88$, pp. 121-150.

MINISTERIO DE MEDIO AMBIENTE RURAL Y MARINO. Gestión Integrada de las Zonas Costeras en España. Disponible en: http://www.magrama.gob.es/es/costas/publicaciones/Informe_GIZC_Spain_enviado_tcm7-30301.pdf.

VV. AA. (2009): Estudios sobre la ordenación, planificación y gestión del litoral: Hacía un modelo integrado y sostenible. (Dir. Francisco Javier Sanz Larruga, y Coord. Marta García Pérez). Fundación Pedro Barrié de la Maza. A Coruña.

FARINÓS DASÍ, J. (Ed. y Coord.) (2011): La gestión integrada de zonas costeras ¿algo más que una ordenación del litoral revisada? La GIZC como evolución de las prácticas de planificación y gobernanza territoriales. Valencia. Universitat de Valencia.

CONSELLERÍA DE MEDIO AMBIENTE, TERRITORIO E INFRAESTRUCTURAS. XUNTA DE GALICIA (2011): Plan de Ordenación del Litoral de Galicia. (Dir. Gral. Manuel Borobio Sanchiz, y Dir. Tec. Miriam García García). Disponible en: http://www. xunta.es/litoral/web/index.php. 